Published in final edited form as:

Cancer Res. 2010 November 15; 70(22): 9218-9223. doi:10.1158/0008-5472.CAN-10-0985.

\title{
Serum Vitamin D and Risk of Bladder Cancer
}

\section{Alison M. Mondul ${ }^{1}$, Stephanie J. Weinstein ${ }^{1}$, Satu Männistö ${ }^{2}$, Kirk Snyder ${ }^{3}$, Ronald L. Horst $^{4}$, Jarmo Virtamo ${ }^{2}$, and Demetrius Albanes ${ }^{1}$}

${ }^{1}$ Nutritional Epidemiology Branch, Division of Cancer Epidemiology and Genetics, National Cancer Institute, $\mathrm{NIH}$, Department of Health and Human Services, Bethesda, Maryland ${ }^{2}$ Department of Chronic Disease Prevention, National Institute for Health and Welfare, Helsinki, Finland ${ }^{3}$ Information Management Services, Inc., Silver Spring, Maryland ${ }^{4}$ Heartland Assays, Inc., Ames, lowa

\section{Abstract}

Vitamin D may protect against several cancers, but data regarding the association between circulating vitamin $\mathrm{D}$ and bladder cancer are limited.

Within the ATBC Study, a randomized controlled trial conducted to determine the effects of $\alpha$ tocopherol and $\beta$-carotene supplements on cancer incidence in male smokers, 250 bladder cancer cases were randomly sampled by month of blood collection. Controls were matched 1:1 to cases on age at randomization and date of blood collection. Conditional logistic regression was used to estimate odds ratios (OR) and $95 \%$ confidence intervals (CI) of bladder cancer by a priori categories of baseline serum 25-hydroxyvitamin D (25(OH)D)(i.e., $<25,25-<37.5,37.5-<50, \geq$ $50 \mathrm{nmol} / \mathrm{L}$ ), and by season-specific quartiles.

After multivariable adjustment, we found lower $25(\mathrm{OH}) \mathrm{D}$ was associated with a statistically significantly increased risk of bladder cancer (OR, $95 \%$ CI vs. $\geq 50 \mathrm{nmol} / \mathrm{L} ;<25 \mathrm{nmol} / \mathrm{L}: 1.85$, 1.11 - 3.08; 25 - <37.5 nmol/L: 1.87, 1.09 - 3.20; 37.5 - <50 nmol/L: 1.81, $1.06-3.08 ; p$ trend $=0.02$ ). Similarly increased risks for the lowest vitamin $\mathrm{D}$ category were observed when season-specific quartiles were used (OR, 95\% CI Q1 vs. Q4: 1.63, $0.96-2.75$, $p$-trend $=0.03)$.

In this prospective study of male smokers, lower serum 25(OH)D was associated with an increased risk of bladder cancer. Future studies should examine the association in other populations, especially non-smokers and women.

\section{Keywords}

Urinary bladder neoplasms; 25-hydroxyvitamin D; Case-control studies

\section{Introduction}

Urinary bladder cancer is an important health concern in developed countries, particularly for men, among whom incidence is $3-5$ times greater than in women $(1,2)$. In the United States, bladder cancer is the fourth most common cancer diagnosed among men, and the eighth leading cause of cancer death (3). Cigarette smoking, occupational exposure to certain chemical carcinogens, and, in the Middle East, infection with Schistosoma hematobium, are known risk factors for bladder cancer $(1,2)$, although they do not fully

Corresponding Author Contact: Alison M. Mondul, 6120 Executive Blvd. Ste. 320, Rockville MD, 21045, mondulam@ mail.nih.gov, phone: (301) 496-5626, fax: (301) 496-6829. 
explain its etiology. Dietary factors, including intake of fruits and vegetables, red meat, and micronutrients such as vitamins $\mathrm{A}, \mathrm{C}$, and $\mathrm{E}$ are hypothesized to influence bladder cancer risk, but evidence remains inconclusive (1).

Vitamin $\mathrm{D}$ is one nutritional factor that is thought to protect against cancer at many sites $(4,5)$. The primary circulating form of vitamin $\mathrm{D}$ is 25 -hydroxyvitamin $\mathrm{D}(25(\mathrm{OH}) \mathrm{D})$, which is considered the best indicatior of an individual's vitamin $\mathrm{D}$ status $(5) .25(\mathrm{OH}) \mathrm{D}$ is converted to its active form, 1-25-dihydroxyvitamin $\mathrm{D}\left(1,25(\mathrm{OH})_{2} \mathrm{D}\right)$, by 1- $\alpha$-hydroxylase $(5,6) .1,25(\mathrm{OH})_{2} \mathrm{D}$ has been shown to promote cell differentiation and decrease proliferation, invasion, angiogenesis, and metastasis $(4,5)$. Because most cells in the body express $1-\alpha$ hydroxylase, it is reasonable to hypothesize that $1,25(\mathrm{OH})_{2} \mathrm{D}$ is available locally and may prevent cancer in multiple organs $(4,5)$, with evidence being supportive for colorectal cancer and suggestive for breast cancer (5). On the other hand, recent results from the Cohort Consortium Vitamin D Pooling Project of rarer cancers, which examined the association between serum vitamin $\mathrm{D}$ and risk of pancreatic, ovarian, upper gastrointestinal, endometrial, and renal cancers, and lymphoma, suggest that vitamin D has little influence on the occurrence of cancer at these sites (7-12). Few studies have examined the association between vitamin $\mathrm{D}$ and bladder cancer, however.

One laboratory study demonstrated vitamin D inhibition of bladder cancer cell proliferation and bladder tumorigenesis in rats (13). To our knowledge, no studies have examined the association between serum vitamin $\mathrm{D}$ and risk of bladder cancer, although two studies of vitamin D intake found no association (14) and an inverse association (only among individuals $\geq 63$ years old) (15). Self-reported dietary intake of vitamin D is a less accurate measure vitamin D status than are serum or tissue $25(\mathrm{OH}) \mathrm{D}$ concentrations, however. One study reported bladder cancer risk to be higher among persons with alleles of the vitamin D receptor rs 10735810 polymorphism ("Fokl”) that are known to decrease the receptor's activity (16).

We analyzed nested case-control data from the Alpha-Tocopherol, Beta-Carotene Cancer Prevention (ATBC) Study, a large primary cancer prevention trial of $\alpha$-tocopherol and $\beta$ carotene supplementation, to examine whether circulating concentration of $25(\mathrm{OH}) \mathrm{D}$ was prospectively associated with risk of bladder cancer.

\section{Materials and Methods}

The ATBC Study was a randomized, double-blind, placebo-controlled, primary prevention trial conducted to determine the effects of supplementation with $\alpha$-tocopherol and $\beta$-carotene on cancer incidence (17). Caucasian male smokers $(n=29,133)$ from southwestern Finland were recruited between 1985 and 1988. Men were between 50-69 years old at baseline and smoked at least 5 cigarettes per day as part of the enrollment criteria. Men were ineligible if they had previously had cancer or another serious illness at enrollment, or if they reported current use of supplements containing vitamin E (>20mg), vitamin A (>20,000 IU), or $\beta$ carotene $(>6 \mathrm{mg})$. Men who were enrolled in the trial were assigned to one of four groups based on a $2 \times 2$ factorial design: 1) $\alpha$-tocopherol (dl- $\alpha$-tocopheryl acetate, $50 \mathrm{mg} /$ day), 2) $\beta$ carotene (20 mg/day), 3) both supplements, or 4) placebo. Trial participants were supplemented for 5-8 years, until death, or until the trial ended on April 30, 1993. Although the intervention trial ended, follow-up is ongoing through the Finnish Cancer Registry and the Register of Causes of Death and for this analysis is complete through April 20, 2005. The ATBC Study was approved by institutional review boards at both the US National Cancer Institute and the Finnish National Public Health Institute, and written informed consent was obtained from all participants. At enrollment, participants completed questionnaires about general risk factors, smoking, and medical history, as well as a food- 
frequency questionnaire. Participants also underwent physical examination by registered nurses to measure their height and weight, and to collect an overnight fasting blood sample.

Bladder cancer cases were identified by linkage with the Finnish Cancer Registry, which provides nearly $100 \%$ complete incident cancer ascertainment in Finland (18). Medical records for the cases diagnosed prior to September 2001 were reviewed by one or two study oncologists to confirm diagnosis and staging, with subsequent cases reviewed by a study physician. Two hundred fifty of the 558 total bladder cancer cases in the ATBC Study were randomly sampled by month of blood collection such that 25 cases were included from each month, with 25 cases total from June to August, because there were few clinic visits during the summer months (and none in July). Controls were sampled without replacement from ATBC Study participants who were alive and cancer free at the time the case was diagnosed and were matched 1:1 with cases on age at randomization ( \pm 1 year) and date of blood collection ( \pm 30 days). Bladder cancer cases were not eligible to be controls.

Fasting serum samples collected at baseline were stored at $-70{ }^{\circ} \mathrm{C} .25(\mathrm{OH}) \mathrm{D}$ was measured by Heartland Assays, Inc (Ames, IA) using the DiaSorin Liaison 25(OH)D TOTAL assay (19). Each batch of samples contained four or six blinded quality control (QC) samples from our study and from standard reference materials provided by the National Institute of Standards and Technology (NIST) at both Level 1 (prepared from "normal" human serum and not altered, $\sim 60 \mathrm{nmol} / \mathrm{L}$ ) and Level 2 (prepared by diluting Level 1, $\sim 35 \mathrm{nmol} / \mathrm{L}$ ) (20). Inter- and intrabatch CVs were as follows: $12.7 \%$ and $9.3 \%$, respectively, for NIST level 1; $13.6 \%$ and $11.0 \%$, respectively, for NIST level $2 ; 12.3 \%$ and $10.5 \%$, respectively, for the ATBC cohort QC samples. Further details of the laboratory and quality control methods are discussed elsewhere (21).

Conditional logistic regression was used to estimate odds ratios and $95 \%$ confidence intervals of bladder cancer by a priori categories of baseline $25(\mathrm{OH}) \mathrm{D}(<25,25-<37.5,37.5$ $-<50,50-<75, \geq 75 \mathrm{nmol} / \mathrm{L}$ ). The association for the $50-<75$ and $\geq 75 \mathrm{nmol} / \mathrm{L}$ groups was similar, so we collapsed these categories. To capture whether the association differed at very low $25(\mathrm{OH}) \mathrm{D}$ levels, we split the $<25$ category into $<15$ and $15-<25$, but the association was similar. Thus, we present our results for baseline serum $25(\mathrm{OH}) \mathrm{D}$ by the previously defined four-level a priori categories. These cutpoints were chosen using clinical definitions for vitamin D deficiency and sufficiency $(6,22,23)$. Further, because these cutpoints are commonly used in research studies $(7-12,24)$, categorizing serum $25(\mathrm{OH}) \mathrm{D}$ in this way enables comparision of our findings with those from other studies. We chose $\geq 50$ $\mathrm{nmol} / \mathrm{L}$ as the referent category because it contains the mean $25(\mathrm{OH}) \mathrm{D}$ level of the U.S. population as determined in the 2000-2004 National Health and Nutrition Examination Survey (25). We evaluated the trend across categories by modeling the median of each category as a continuous variable and evaluating its statistical significance using the Wald test. Because bladder cancer is a rare disease, the odds ratio is a good approximation of the relative risk, and we use these terms interchangeably. All models were conditioned on the matching factors (age and date of blood collection).

The following factors that are hypothesized or known to be associated with either bladder cancer or vitamin D were assessed as potential confounding variables: height, weight, BMI, urban residence, attained education, skin behavior in the sun, trips to the south, cigarettes per day, years smoked, family history of bladder cancer, physical activity, marital status, high-risk occupation (chemical dye manufacture), intake of dairy, total fluid, dietary calcium, supplemental vitamin D, and supplemental calcium, and serum total cholesterol, $\alpha$ tocopherol, $\beta$-carotene, and retinol. Although family history of bladder cancer and high-risk occupation appeared to be associated with the outcome, there were too few individuals in these groups (family history, $n=6$, and high-risk occupation, $n=8$ ) to adjust for them in the 
multivariable model. Each of the remaining variables was entered into the age-adjusted model to evaluate whether the point estimates for $25(\mathrm{OH}) \mathrm{D}$ categories changed by at least $10 \%$, and none did so. Thus, our final model is conditioned on the matching factors (age and date of blood collection) without adjustment for further covariates. We also present our main results further adjusted for cigarettes per day and years smoked because smoking is such a strong, well-established risk factor for bladder cancer.

Because 25(OH)D levels are known to vary by season we used two approaches to address its influence. First, as mentioned previously, blood was collected throughout the year (except in July) and cases and controls were matched on date of blood draw. Second, we examined the bladder cancer association by categorizing men according to season of blood draw-specific quartiles of $25(\mathrm{OH}) \mathrm{D}$, based on the control distribution of each season (sunnier season $=$ June - October, darker season $=$ November - May). Analyses were conducted stratifying by age at diagnosis ( $<60, \geq 60$ years), cigarettes smoked per day ( $<20, \geq 20)$, $\alpha$-tocopherol and $\beta$-carotene intervention arms, season (sunnier season, darker season), physical activity (yes $=$ at least light or moderate occupational activity or moderate leisure time activity), time between blood collection and case diagnosis $(<10, \geq 10$ years), and less than median versus $\geq$ median of BMI, serum total cholesterol, and dietary intake of vitamin D and calcium. Stratified analyses were conducted using unconditional logistic regression adjusting for the matching factors. The main model results were unchanged when this approach was used instead of conditional logistic regression, making biased estimates unlikely. Statistical interaction was assessed using the likelihood ratio test.

\section{Results}

Characteristics of cases and controls were essentially similar with the exception of heavier smoking and somewhat lower consumption of poultry among cases (Table 1).

Lower serum 25(OH)D was associated with a statistically significantly increased risk of bladder cancer (Table 2). Men in each of the three categories of $25(\mathrm{OH}) \mathrm{D}$ below $50 \mathrm{nmol} / \mathrm{L}$ were at nearly twice the risk compared to men with $25(\mathrm{OH}) \mathrm{D} \geq 50 \mathrm{nmol} / \mathrm{L},(\mathrm{OR}, 95 \% \mathrm{CI}$ $<50 \mathrm{nmol} / \mathrm{L}$ vs. $\geq 50 \mathrm{nmol} / \mathrm{L}=1.84,1.20-2.84, p$-value $=0.005)$. The results were somewhat attenuated with adjustment for smoking (Table 2). We observed similar results when quartiles based on season of blood draw were used (Table 2). For every $1 \mathrm{nmol} / \mathrm{L}$ increase in $25(\mathrm{OH}) \mathrm{D}$, the odds of bladder cancer decreased by $2.4 \%(p=0.04)$.

There was no statistical evidence for interactions with any of the a priori factors examined (data not shown). There was a suggestion, however, that the vitamin D -bladder cancer association was stronger among men whose blood was drawn in the sunnier months, who were not receiving the trial $\alpha$-tocopherol supplement, and who were more physically active, and for bladder cancer cases diagnosed within 10 years of blood collection (Table 3 ). These interactions were not statistically significant, however.

\section{Discussion}

We observed that men with lower $25(\mathrm{OH}) \mathrm{D}$ serum concentrations were at increased risk of bladder cancer compared to men with higher serum levels. These findings are consistent with previous cell culture, in vivo, and genetic evidence suggesting that greater exposure to vitamin D could have a role in protecting against bladder cancer $(13,15,16)$.

Our results were somewhat attenuated, but more consistent with a dose-risk association, when we classified men based on season-specific vitamin D categories, as compared with a priori cutpoints. Which method most accurately accounts for normal seasonal fluctuation in vitamin D levels is not clear. One recent simulation study suggested that use of season- 
specific categories yielded less biased risk estimates compared with adjustment for season through a covariate term in multivariable models, although the influence of case-control matching on date of blood collection, as was done in the present study, was not evaluated (26). It is important to note, however, that regardless of how vitamin D was categorized in our analysis, men with lower vitamin D levels were at substantially increased risk of bladder cancer, with the OR range of 1.6-1.7 for the lowest categories being quite consistent across approaches. Interestingly, although the interaction test was not statistically significant and the observation could be due to chance, the inverse association appeared stronger among men whose blood was obtained during the sunnier months. If substantiated by other studies, this could indicate that lower circulating vitamin $\mathrm{D}$ during periods expected to reflect greater solar UVB-related vitamin D biosynthesis is a more sensitive predictor of bladder cancer risk than are lower levels obtained during the darker, "basal-state" months. Our data also indicate that the association between vitamin $\mathrm{D}$ status and risk of bladder cancer might be stronger in men whose blood was collected less than 10 years prior to diagnosis. One interpretation of this finding is that lower vitamin D status may play a greater role later, rather than earlier, in bladder carcinogenesis; i.e., by promoting tumor growth and progression. Although this is speculative and requires examination in other cohorts with long follow-up, it is consistent with experimental findings of cancer cell culture growth inhibition by $1,25(\mathrm{OH})_{2} \mathrm{D}(13)$.

Our measurement of 25(OH)D in prospectively-collected serum in one laboratory, and our detailed information on potential confounding factors, including date of blood draw, smoking dose and duration, physical activity, and dietary and supplemental intakes of vitamin $\mathrm{D}$, are important strengths of this study. Although we were able to examine many potential effect modifiers, we had limited statistical power to detect modest differences between strata. It is possible that larger studies could clarify the suggested interactions observed here; i.e., with season of blood draw, physical activity, and vitamin $\mathrm{E}$ supplementation.

In this population of male smokers, lower serum $25(\mathrm{OH}) \mathrm{D}$ was associated with an increased risk of bladder cancer over a 20 year period. Future studies should examine the association in other populations, especially non-smokers and women, and evaluate possible effect modification by season of blood draw, physical activity, and intake of other nutrients, including vitamin $\mathrm{E}$.

\section{Acknowledgments}

This work was was supported by U.S. Public Health Service (contract numbers N01-CN-45165, N01-RC-45035, N01-RC-37004, and HHSN261201000006C) from the National Cancer Institute, Department of Health and Human Services, and by funding from the Intramural Research Program of the National Cancer Institute.

\section{References}

1. Brinkman M, Zeegers MP. Nutrition, total fluid and bladder cancer. Scand J Urol Nephrol Suppl. 2008:25-36. [PubMed: 18815914]

2. Parkin DM. The global burden of urinary bladder cancer. Scand J Urol Nephrol Suppl. 2008:12-20. [PubMed: 19054893]

3. Jemal A, Siegel R, Ward E, Hao Y, Xu J, Thun MJ. Cancer statistics, 2009. CA Cancer J Clin. 2009; 59:225-49. [PubMed: 19474385]

4. Holick MF. Vitamin D: importance in the prevention of cancers, type 1 diabetes, heart disease, and osteoporosis. Am J Clin Nutr. 2004; 79:362-71. [PubMed: 14985208]

5. Giovannucci E. The epidemiology of vitamin D and cancer incidence and mortality: a review (United States). Cancer Causes Control. 2005; 16:83-95. [PubMed: 15868450] 
6. Holick MF. Vitamin D status: measurement, interpretation, and clinical application. Ann Epidemiol. 2009; 19:73-8. [PubMed: 18329892]

7. Abnet CC, Chen Y, Chow WH, et al. Circulating 25-hydroxyvitamin D and risk of esophageal and gastric cancer: Cohort Consortium Vitamin D Pooling Project of Rarer Cancers. Am J Epidemiol. 172:94-106. [PubMed: 20562192]

8. Gallicchio, L.; Moore, LE.; Stevens, VL., et al. Am J Epidemiol. Vol. 172. Circulating 25hydroxyvitamin D and risk of kidney cancer: Cohort Consortium Vitamin D Pooling Project of Rarer Cancers; p. 47-57.

9. Purdue MP, Freedman DM, Gapstur SM, et al. Circulating 25-hydroxyvitamin D and risk of nonhodgkin lymphoma: Cohort Consortium Vitamin D Pooling Project of Rarer Cancers. Am J Epidemiol. 172:58-69. [PubMed: 20562184]

10. Stolzenberg-Solomon RZ, Jacobs EJ, Arslan AA, et al. Circulating 25-hydroxyvitamin D and risk of pancreatic cancer: Cohort Consortium Vitamin D Pooling Project of Rarer Cancers. Am J Epidemiol. 172:81-93. [PubMed: 20562185]

11. Zeleniuch-Jacquotte A, Gallicchio L, Hartmuller V, et al. Circulating 25-hydroxyvitamin D and risk of endometrial cancer: Cohort Consortium Vitamin D Pooling Project of Rarer Cancers. Am J Epidemiol. 172:36-46. [PubMed: 20562189]

12. Zheng W, Danforth KN, Tworoger SS, et al. Circulating 25-hydroxyvitamin D and risk of epithelial ovarian cancer: Cohort Consortium Vitamin D Pooling Project of Rarer Cancers. Am J Epidemiol. 172:70-80. [PubMed: 20562186]

13. Konety BR, Lavelle JP, Pirtskalaishvili G, et al. Effects of vitamin D (calcitriol) on transitional cell carcinoma of the bladder in vitro and in vivo. J Urol. 2001; 165:253-8. [PubMed: 11125420]

14. Michaud DS, Spiegelman D, Clinton SK, Rimm EB, Willett WC, Giovannucci E. Prospective study of dietary supplements, macronutrients, micronutrients, and risk of bladder cancer in US men. Am J Epidemiol. 2000; 152:1145-53. [PubMed: 11130620]

15. Brinkman MT, Karagas MR, Zens MS, Schned A, Reulen RC, Zeegers MP. Minerals and vitamins and the risk of bladder cancer: results from the New Hampshire Study. Cancer Causes Control. 2009

16. Mittal RD, Manchanda PK, Bhat S, Bid HK. Association of vitamin-D receptor (Fok-I) gene polymorphism with bladder cancer in an Indian population. BJU Int. 2007; 99:933-7. [PubMed: 17378851]

17. The ATBC Cancer Prevention Study Group. The alpha-tocopherol, beta-carotene lung cancer prevention study: design, methods, participant characteristics, and compliance. Ann Epidemiol. 1994; 4:1-10. [PubMed: 8205268]

18. Korhonen P, Malila N, Pukkala E, Teppo L, Albanes D, Virtamo J. The Finnish Cancer Registry as follow-up source of a large trial cohort--accuracy and delay. Acta Oncol. 2002; 41:381-8. [PubMed: 12234031]

19. Ersfeld DL, Rao DS, Body JJ, et al. Analytical and clinical validation of the $25 \mathrm{OH}$ vitamin D assay for the LIAISON automated analyzer. Clin Biochem. 2004; 37:867-74. [PubMed: 15369717]

20. (NIST) NIoSaT. Certificate of Analysis, Standard Reference Matiral 972 Vitamin D in Human Serum. 2009. [cited 2009; Available from: https://www-s.nist.gov/srmors/view_detail.cfm?srm=972

21. Gallicchio L, Helzlsouer KJ, Chow WH, et al. Circulating 25-hydroxyvitamin D and the risk of rarer cancers: Design and methods of the Cohort Consortium Vitamin D Pooling Project of Rarer Cancers. Am J Epidemiol. 172:10-20. [PubMed: 20562188]

22. Holick MF. Vitamin D deficiency. N Engl J Med. 2007; 357:266-81. [PubMed: 17634462]

23. Looker AC, Dawson-Hughes B, Calvo MS, Gunter EW, Sahyoun NR. Serum 25-hydroxyvitamin D status of adolescents and adults in two seasonal subpopulations from NHANES III. Bone. 2002; 30:771-7. [PubMed: 11996918]

24. Jenab M, Bueno-de-Mesquita HB, Ferrari P, et al. Association between pre-diagnostic circulating vitamin D concentration and risk of colorectal cancer in European populations:a nested casecontrol study. BMJ. 340:b5500. 
25. Looker AC, Pfeiffer CM, Lacher DA, Schleicher RL, Picciano MF, Yetley EA. Serum 25-

hydroxyvitamin D status of the US population: 1988-1994 compared with 2000-2004. Am J Clin Nutr. 2008; 88:1519-27. [PubMed: 19064511] 
Table 1

Selected baseline characteristics [medians (interquartile range) or number (percent)] for case and control subjects, ATBC Study

\begin{tabular}{|c|c|c|}
\hline Characteristic & Controls $(n=250)$ & Cases $(n=250)$ \\
\hline Age (years) & $59(56-63)$ & $59(55-63)$ \\
\hline Height (cm) & $173(168-176)$ & $174(169-178)$ \\
\hline Weight (kg) & $76.5(70.1-85.2)$ & $76.5(70.0-86.0)$ \\
\hline BMI $\left(\mathrm{kg} / \mathrm{m}^{2}\right)$ & $25.8(23.5-28.4)$ & $25.5(23.3-27.8)$ \\
\hline Cigarettes per day & $20(15-25)$ & $20(15-25)$ \\
\hline Years of smoking & $38(32-43)$ & $40(35-44)$ \\
\hline \multicolumn{3}{|l|}{ Occupational physical activity } \\
\hline Not working & $125(50.0)$ & $113(45.2)$ \\
\hline Very light/Light & $72(28.8)$ & $85(34.0)$ \\
\hline Moderate/Heavy & $53(21.2)$ & $52(20.8)$ \\
\hline \multicolumn{3}{|l|}{ Physically active } \\
\hline No & 205 & 203 \\
\hline Yes & 45 & 47 \\
\hline Urban residence & $146(58.4)$ & $161(64.4)$ \\
\hline Married & $218(87.2)$ & $215(86.0)$ \\
\hline > Elementary school education & $46(18.4)$ & $59(23.6)$ \\
\hline Family history of bladder cancer & $2(0.9)$ & $4(2.3)$ \\
\hline \multicolumn{3}{|l|}{ Skin behavior in the sun ${ }^{\dagger}$} \\
\hline Burns easily & $76(32)$ & $69(35)$ \\
\hline Burns slightly & $70(29)$ & $48(24)$ \\
\hline Will not burn & $93(39)$ & $80(41)$ \\
\hline Total energy intake (kcal/day) & $2,650(2,241-3,068)$ & $2,597(2,144-3,022)$ \\
\hline Dietary vitamin D (IU/day) & $4.8(3.5-6.9)$ & $4.6(3.0-6.5)$ \\
\hline Dietary calcium (mg/day) & $1,313(1,034-1,737)$ & $1,326(1,017-1,645)$ \\
\hline Dairy (g/day) & $777(553-1,002)$ & $740(507-1,001)$ \\
\hline Poultry (g/day) & $15.7(8.2-27.4)$ & $13.9(8.5-21.3)$ \\
\hline Ethanol (g/day) & $8.5(1.8-23.0)$ & $7.6(1.3-22.7)$ \\
\hline Total beverages (g/day) & $1,972(1,659-2,421)$ & $1,915(1,657-2,304)$ \\
\hline \multicolumn{3}{|l|}{ Supplemental vitamin D use } \\
\hline Yes & $14(5.6)$ & $13(5.8)$ \\
\hline \multicolumn{3}{|l|}{ Supplemental calcium use } \\
\hline Yes & $22(8.8)$ & $23(9.2)$ \\
\hline Serum cholesterol (mmol/L) & $6.1(5.5-6.9)$ & $6.3(5.5-7.0)$ \\
\hline Serum alpha-tocopherol (mg/L) & $11.4(10.2-13.8)$ & $11.6(9.8-14.3)$ \\
\hline Serum beta-carotene $(\mu \mathrm{g} / \mathrm{L})$ & $180(118-284)$ & $185(127-285)$ \\
\hline Serum retinol $(\mu \mathrm{g} / \mathrm{L})$ & $564(498-667)$ & $579(494-666)$ \\
\hline
\end{tabular}

${ }^{\dagger}$ Among the 197 cases and 239 controls that completed the study follow-up form. 


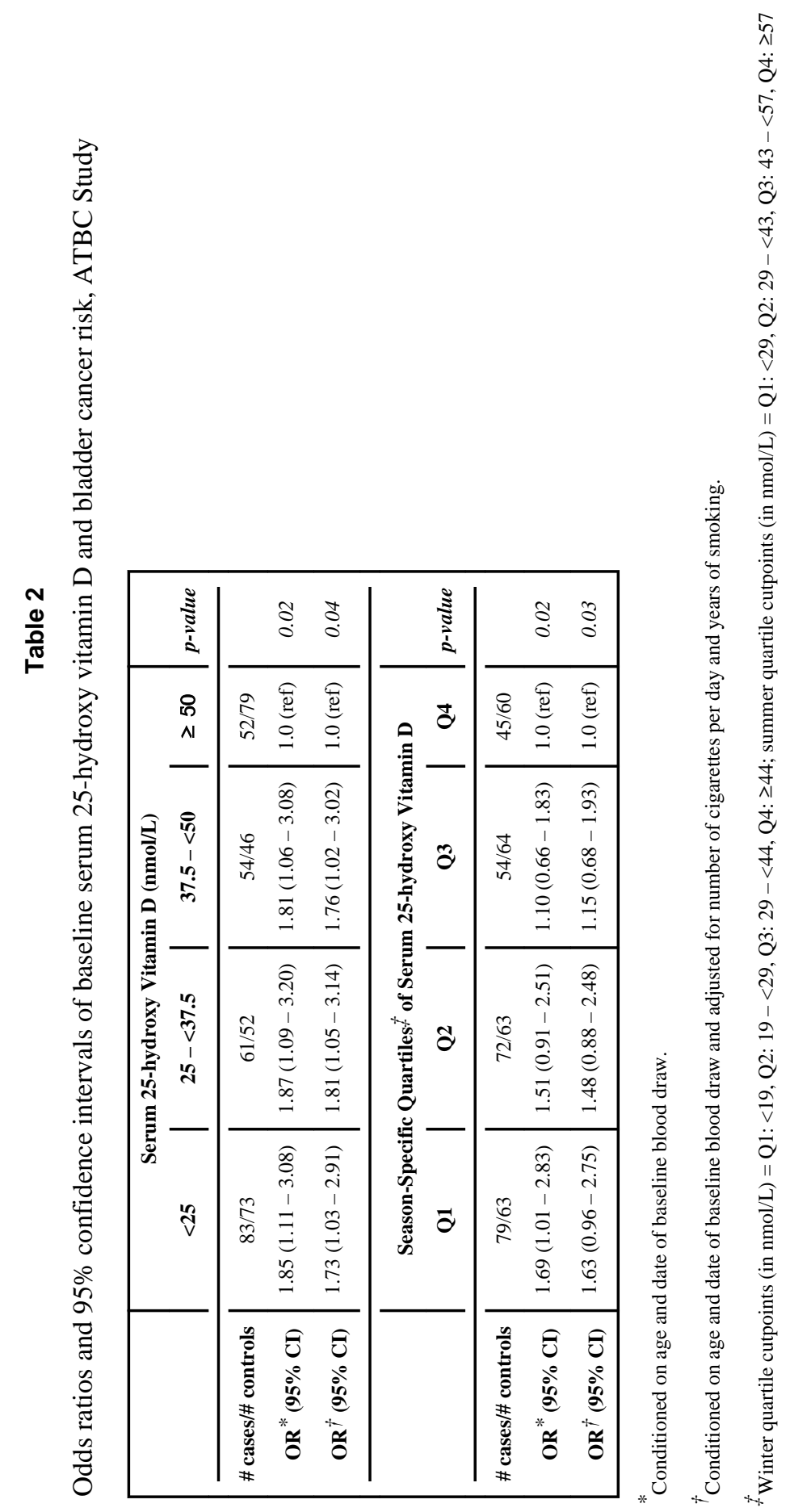


